Supporting information for

\title{
Novel geminal dinitro esters as energetic plasticizers for GAP binder
}

Yinglei Wang ${ }^{a, b^{*}}$, Yajing Liu a, Baodong Zhao a , Fulei Gao a, Weixiao Liu a and Bin

Chen $^{\text {a }}$

a Xi'an Modern Chemistry Research Institute, Xi'an 710065, China

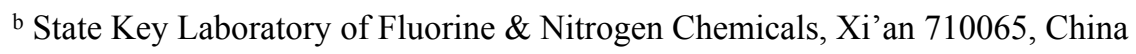

E-mail: wangy1204@163.com.

\section{EXPERIMENTAL SECTION}

General methods. All chemical reagents and solvents were purchased from SigmaAldrich, Acros Organics or ABCR in analytical grade and used without further purification. Purification by column chromatography was performed using Merck silica gel 60 (Ø 35-70 mm).

${ }^{1} \mathrm{H}$ NMR and ${ }^{13} \mathrm{C}$ NMR spectra were recorded using a Bruker-500 MHz instrument and the chemical shifts are given relative to tetramethylsilane as reference material. Infrared spectra was measured with a Perkin Elmer FT-IR 1605 spectrophotometer in the range of 400 to $4000 \mathrm{~cm}^{-1}$ wavelength numbers. Elemental analysis $(\mathrm{C} / \mathrm{H} / \mathrm{N})$ was performed using a Carlo Erba 1106 full-automatic trace element analyzer. Density was determined at room temperature by employing a Micromeritics AccuPyc II 1340 gas pycnometer with a precision syringe $(50 \mu \mathrm{L})$ and a micro balance. Thermal decomposition temperature was determined by a NETZSCH differential scanning calorimeter DSC 204, kinetic analysis details and calculation results were provided in the Supporting Information. The impact sensitivity tests were carried out according to STANAG 4489 using a BAM (Bundesanstalt für Materialforschung) drophammer. The viscosity was recorded using a BROOKFIELD CAP 2000+ viscometer. The glass transition temperature was characterized with a NETZSCH differential scanning calorimeter DSC 200 F3. 
Preparation of 2, 2-dinitropropanol (DNPOH). ${ }^{1,2}$ Nitroethane (15 g, $0.2 \mathrm{~mol}$ ) and $38.5 \%$ aqueous formaldehyde $(15.6 \mathrm{~g}, 0.2 \mathrm{~mol})$ were added into a $250 \mathrm{~mL}$ four-necked round-bottomed flask equipped with a thermometer, a condenser and mechanical stirrer. Then the mixture was cooled down to $-5{ }^{\circ} \mathrm{C}-0{ }^{\circ} \mathrm{C}$ under the ice-bath. Afterwards, the solution of sodium hydroxide $(8 \mathrm{~g}, 0.2 \mathrm{~mol})$ in $40 \mathrm{~mL}$ water was added slowly into the flask with the temperature maintained at $-5{ }^{\circ} \mathrm{C}-0{ }^{\circ} \mathrm{C}$, and the reaction mixture was stirred for $1 \mathrm{~h}$, then the solution of sodium nitrite $(13.8 \mathrm{~g}, 0.2 \mathrm{~mol})$ in 20 $\mathrm{mL}$ water was added slowly into the flask, and stirred for $30 \mathrm{~min}$ under $0{ }^{\circ} \mathrm{C}$. Subsequently, the above-mentioned reaction mixture was added into solution of silver nitrate $(68.0 \mathrm{~g}, 0.4 \mathrm{~mol})$ in $60 \mathrm{~mL}$ water, white precipitate was observed immediately, then the white precipitate changed into black precipitate with metal luster. When the addition was finished, stirring was kept for another $2 \mathrm{~h}$ around $25{ }^{\circ} \mathrm{C}$. Finally, the reaction mixture was filtered, and the filtrate was extracted with dichloromethane (40 $\mathrm{mL} \times 3$ ), and the organic layer was dried with anhydrous magnesium sulfate overnight. After the evaporation of the solvent at reduced pressure, white solid as DNPOH was afforded (21.0 g, yield 70.0 \%). The HPLC purity of the obtained DNPOH was 99.1\%, and was used later without further purification. ${ }^{1} \mathrm{H}$ NMR $(500 \mathrm{MHz}$, acetone, $\delta \mathrm{ppm})$ : 5.35 (t, 1H, OH), $4.46\left(\mathrm{~m}, 2 \mathrm{H}, \mathrm{CH}_{2}\right), 2.25\left(\mathrm{~m}, 3 \mathrm{H}, \mathrm{CH}_{3}\right)$; IR (KBr): 3564, $3427(-\mathrm{OH})$, 2956, 2891, 1451, $1409\left(-\mathrm{CH}_{2}\right), 1567,1333\left(-\mathrm{NO}_{2}\right), 1071(\mathrm{C}-\mathrm{O}) \mathrm{cm}^{-1}$; Elemental analysis calculated for $\mathrm{C}_{3} \mathrm{H}_{6} \mathrm{~N}_{2} \mathrm{O}_{5}$ (150.09): C 24.01, $\mathrm{H}$ 4.03, $\mathrm{N}$ 18.66; found $\mathrm{C} 23.81$, H 4.06, N 18.55.

Thermal kinetic evaluation. Thermal decomposition kinetics investigation along with thermal analysis of energetic materials are important to better understand their decomposition mechanism as well as long term stability assessment involved in handling, storage, and processing stages. Therefore, thermal decomposition kinetics of the DNEPs were further explored preliminarily to obtain its activation energy of thermal decomposition. In order to obtain the kinetic parameters (the apparent activation energy $(E)$ and pre-exponential constant $(A))$ of the exothermic decomposition reaction of the DNEPs, multiple heating methods including Kissinger's method ${ }^{3}$ and Ozawa's method ${ }^{4}$ were employed as follows: 
Kissinger's equation:

$\ln \left(\frac{\beta}{T_{\mathrm{p}}^{2}}\right)=\ln \frac{A R}{E}-\frac{E}{R T_{\mathrm{p}}}$

Ozawa's equation:

$$
\ln (\beta)=-1.0516 \frac{E}{R T_{\mathrm{p}}}+\text { const. }
$$

where $T_{\mathrm{p}}$ is the peak temperature $(\mathrm{K}), \beta$ is the linear heating rate $\left(\mathrm{K} \cdot \mathrm{min}^{-1}\right), E$ is the apparent activation energy $\left(\mathrm{kJ} \cdot \mathrm{mol}^{-1}\right), A$ is the pre-exponential constant $\left(\mathrm{s}^{-1}\right), R$ is the gas constant $\left(\mathrm{J} \cdot \mathrm{mol}^{-1} \cdot \mathrm{K}^{-1}\right)$ and $C$ is a constant. Kinetic plots for DNEPs using the Kissinger and Ozawa methods were depicted in Figures S4-S6, and the calculated results were summarized in Tables S1-S3.

References

(1) Bricker C. E. Synthesis of geminal dinitro compounds. US 696680, 1985.

(2) Garver, L.; Grakauskas, V.; Baum, K. Catalyzed Oxidative Nitration of Nitronate Salts. J. Org. Chem. 1985, 50, 1699-1702.

(3) Kissinger H. E. Reaction Kinetics in Differential Thermal Analysis. Anal. Chem., 1957, 29, 1702-1706.

(4) Ozawa T. A new method of analyzing thermogravimetric data. Bull. Chem. Soc. Jpn., 1957, 38, 1881-1886. 


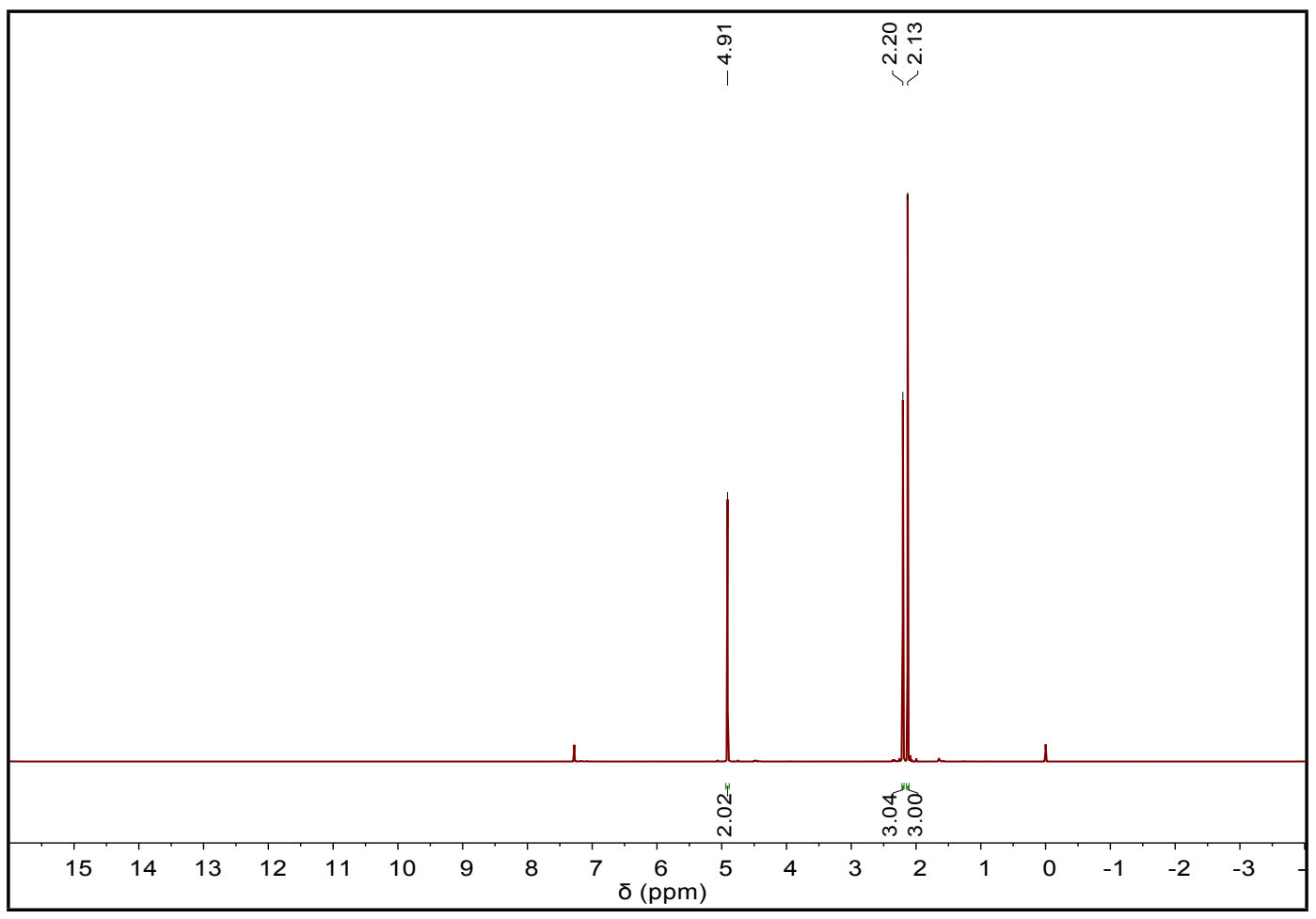

(a) ${ }^{1} \mathrm{H}$ NMR spectrum of DNPAc

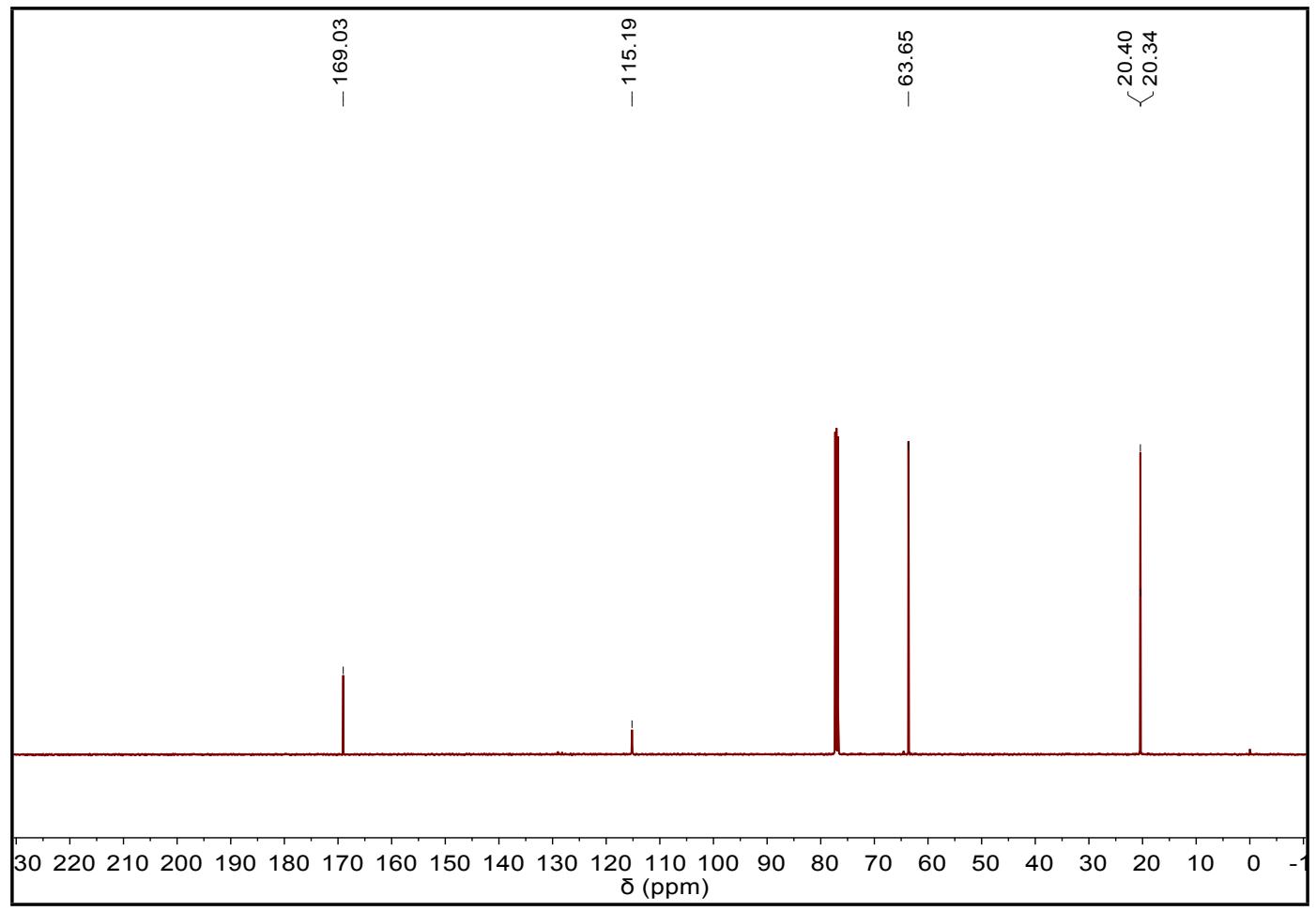

(b) ${ }^{13} \mathrm{C}$ NMR spectrum of DNPAc 
Figure S1 (a) ${ }^{1} \mathrm{H}$ NMR and (b) ${ }^{13} \mathrm{C}$ NMR spectra of DNPAc.

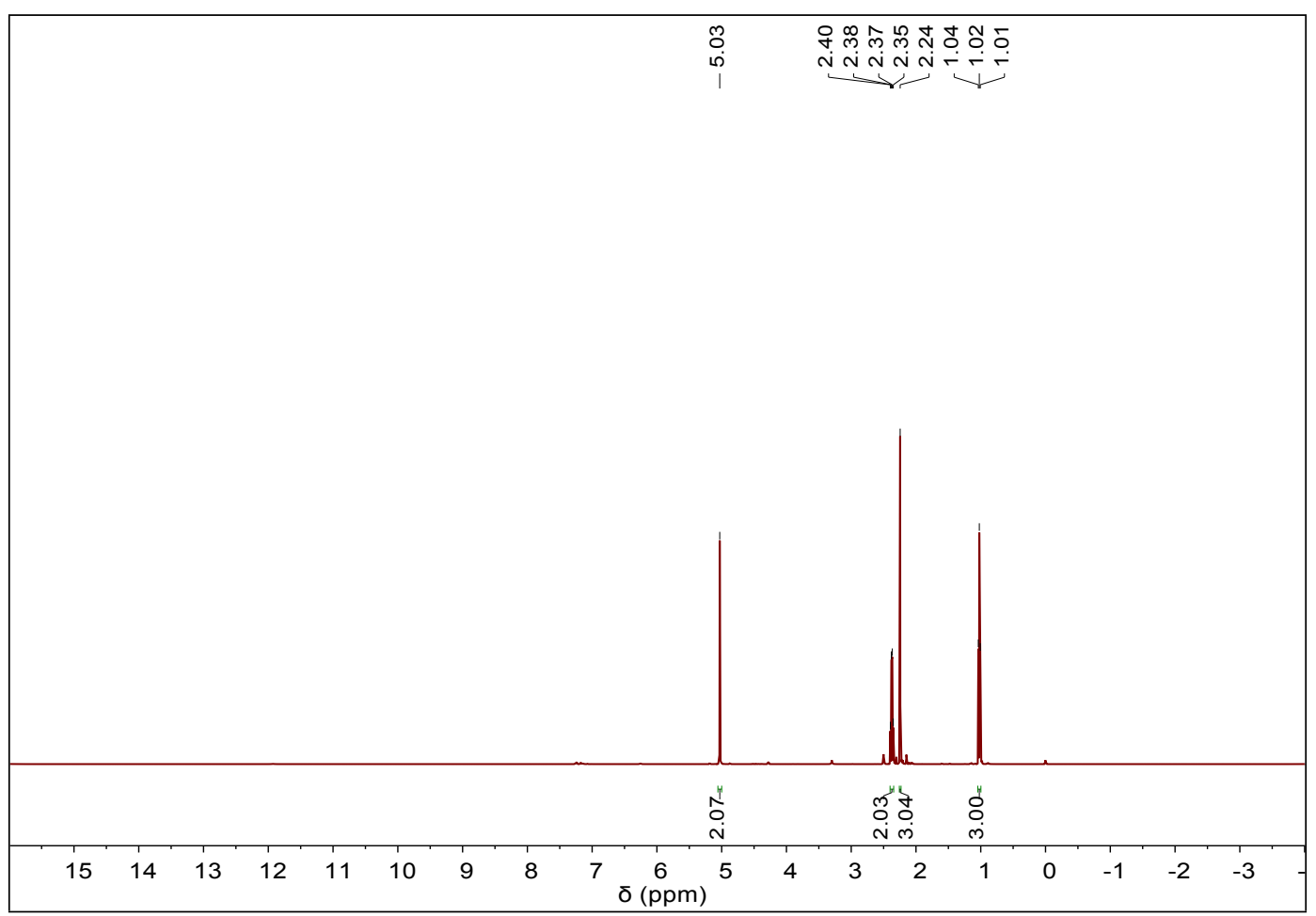

(a) ${ }^{1} \mathrm{H}$ NMR spectrum of DNPPc

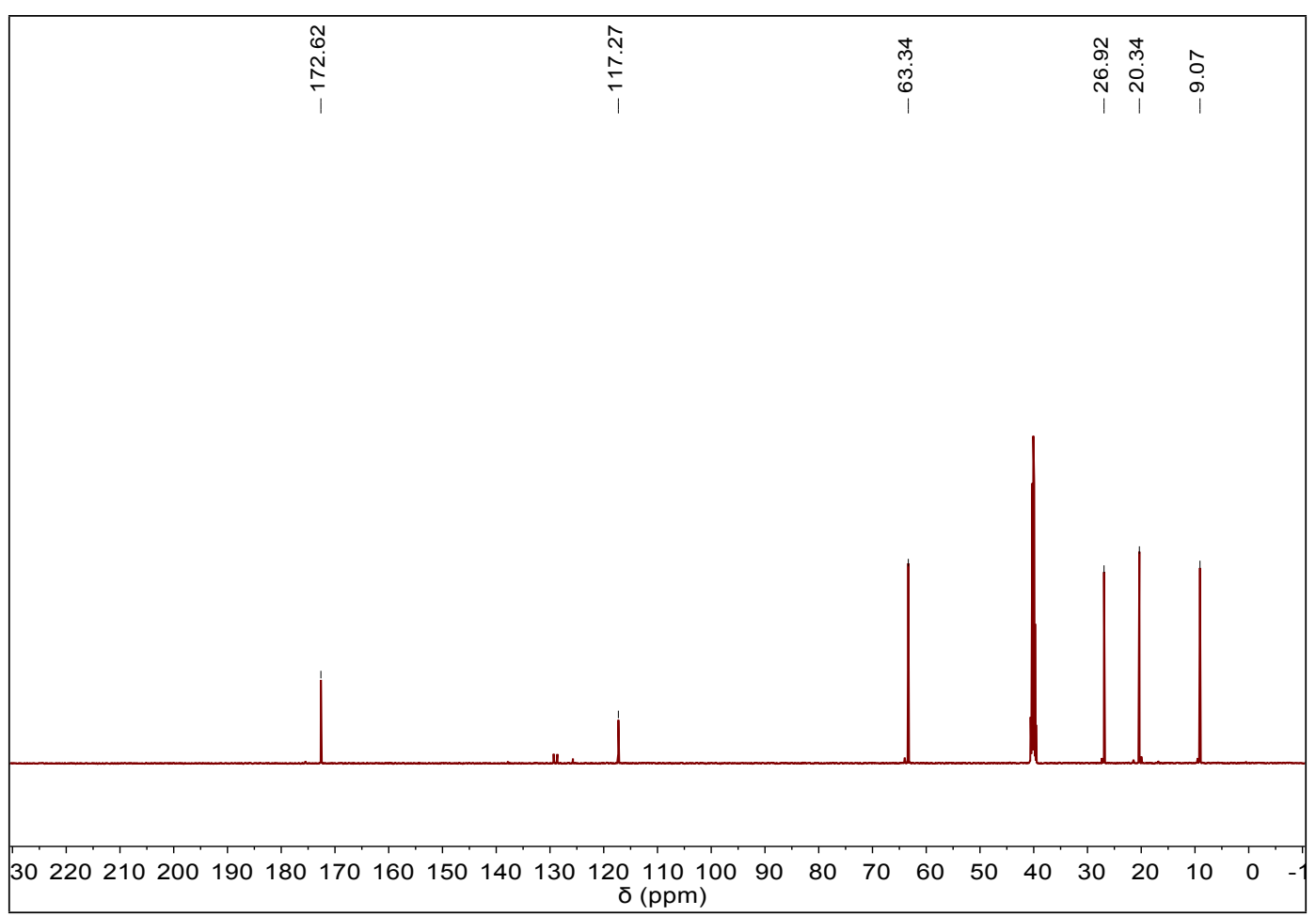

(b) ${ }^{13} \mathrm{C}$ NMR spectrum of DNPPc 
Figure S2 (a) ${ }^{1} \mathrm{H}$ NMR and (b) ${ }^{13} \mathrm{C}$ NMR spectra of DNPPc.

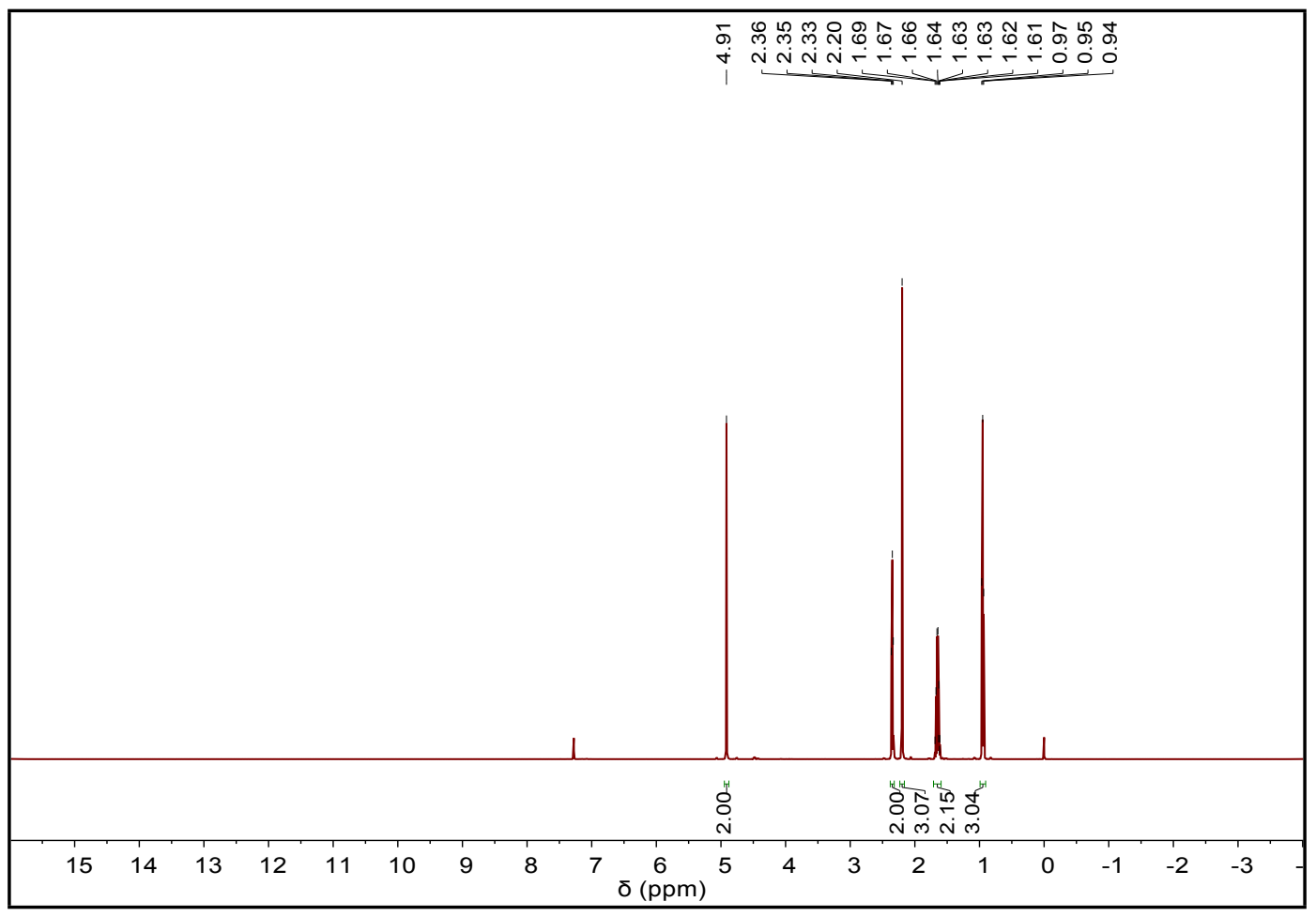

(a) ${ }^{1} \mathrm{H}$ NMR spectrum of DNPBc

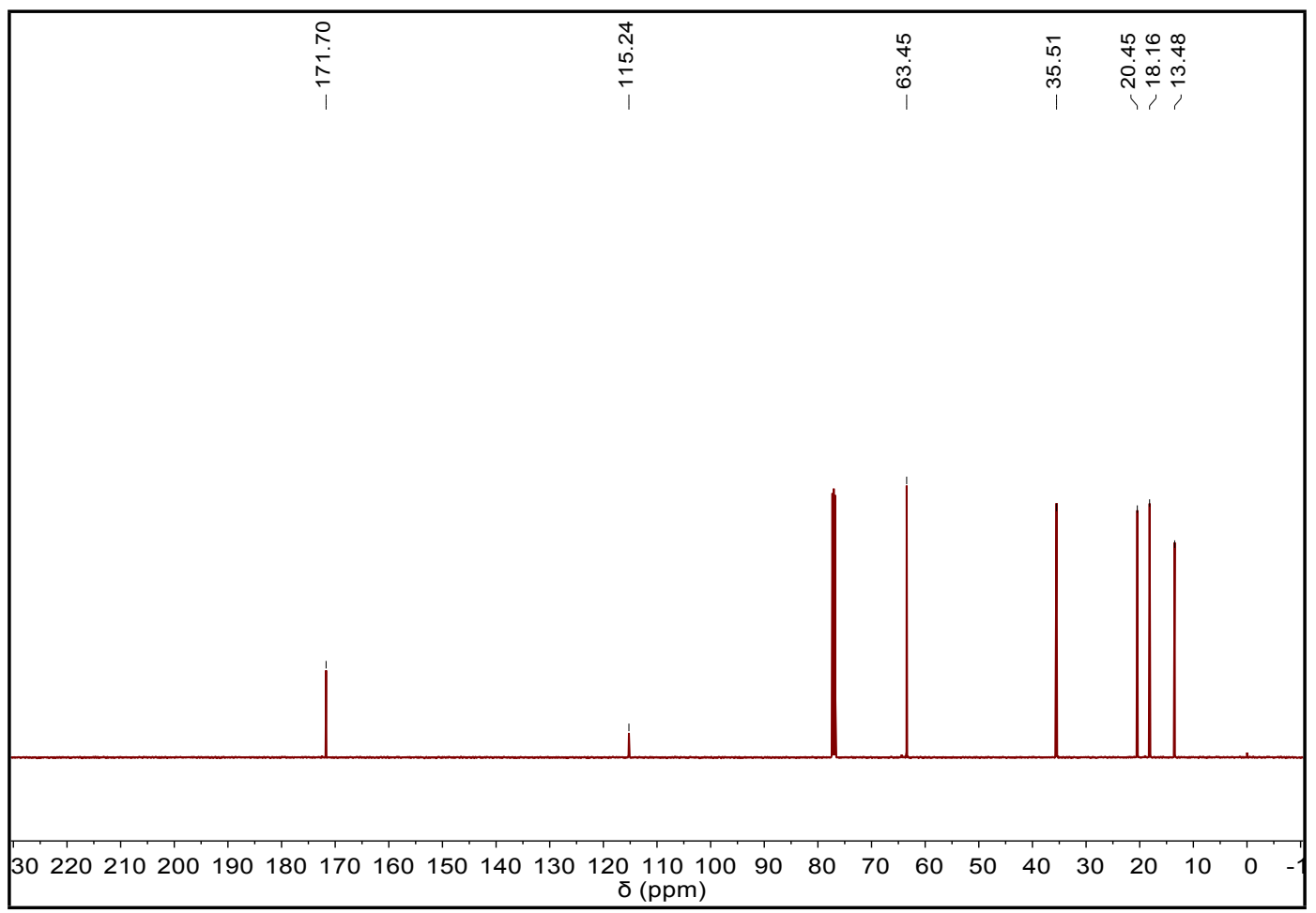

(b) ${ }^{13} \mathrm{C}$ NMR spectrum of DNPBc 
Figure S3 (a) ${ }^{1} \mathrm{H}$ NMR and (b) ${ }^{13} \mathrm{C}$ NMR spectra of DNPBc.

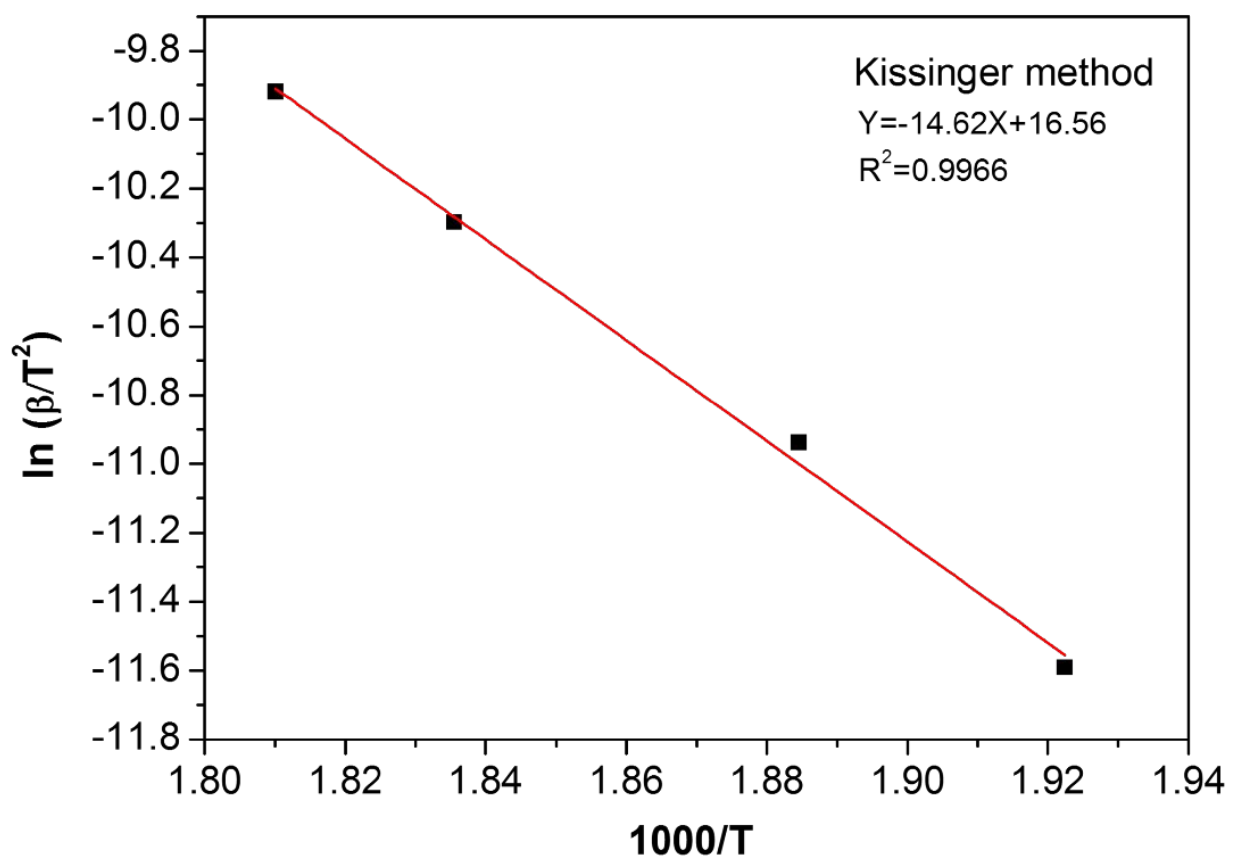

(a) Kinetic plot for DNPAc by Kissinger method

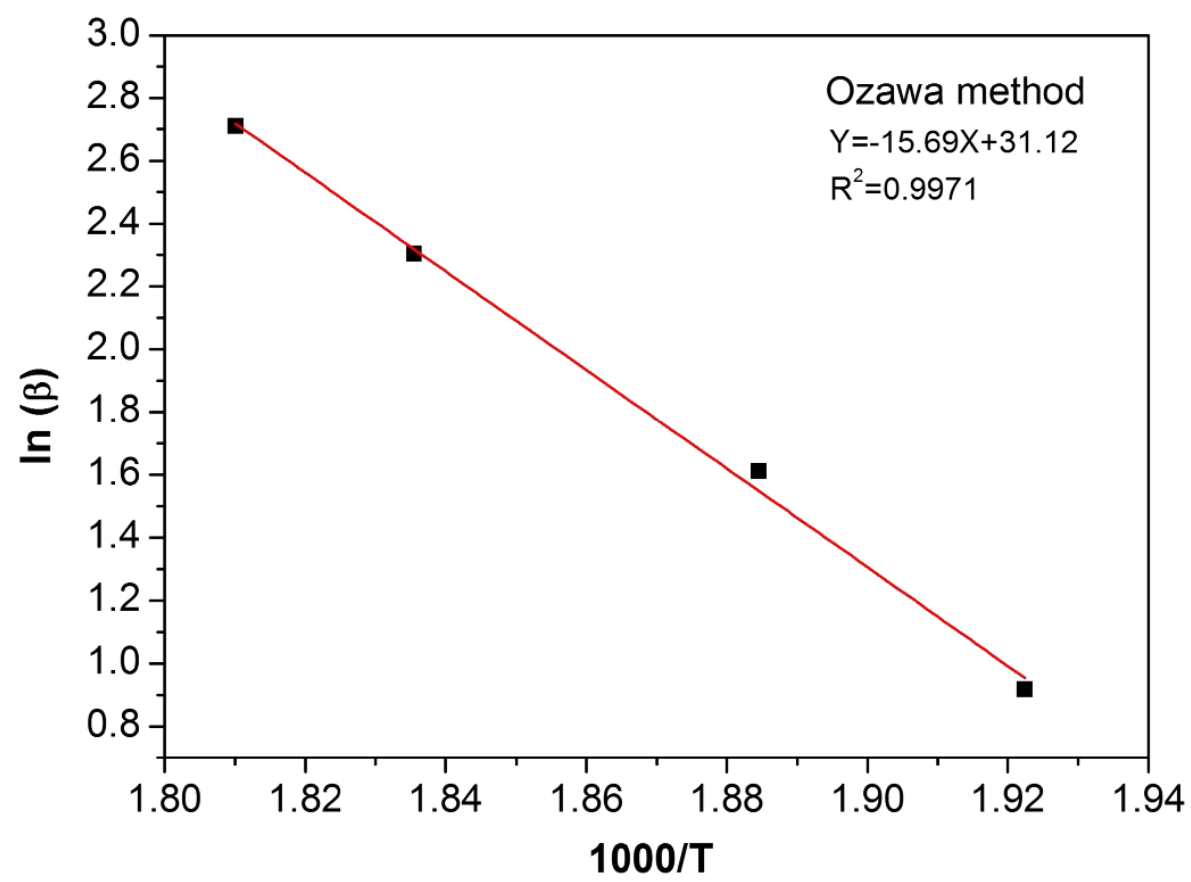

(b) Kinetic plot for DNPAc by Ozawa method 
Figure S4 Kinetic plots for DNPAc by (a) Kissinger method and (b) Ozawa method.

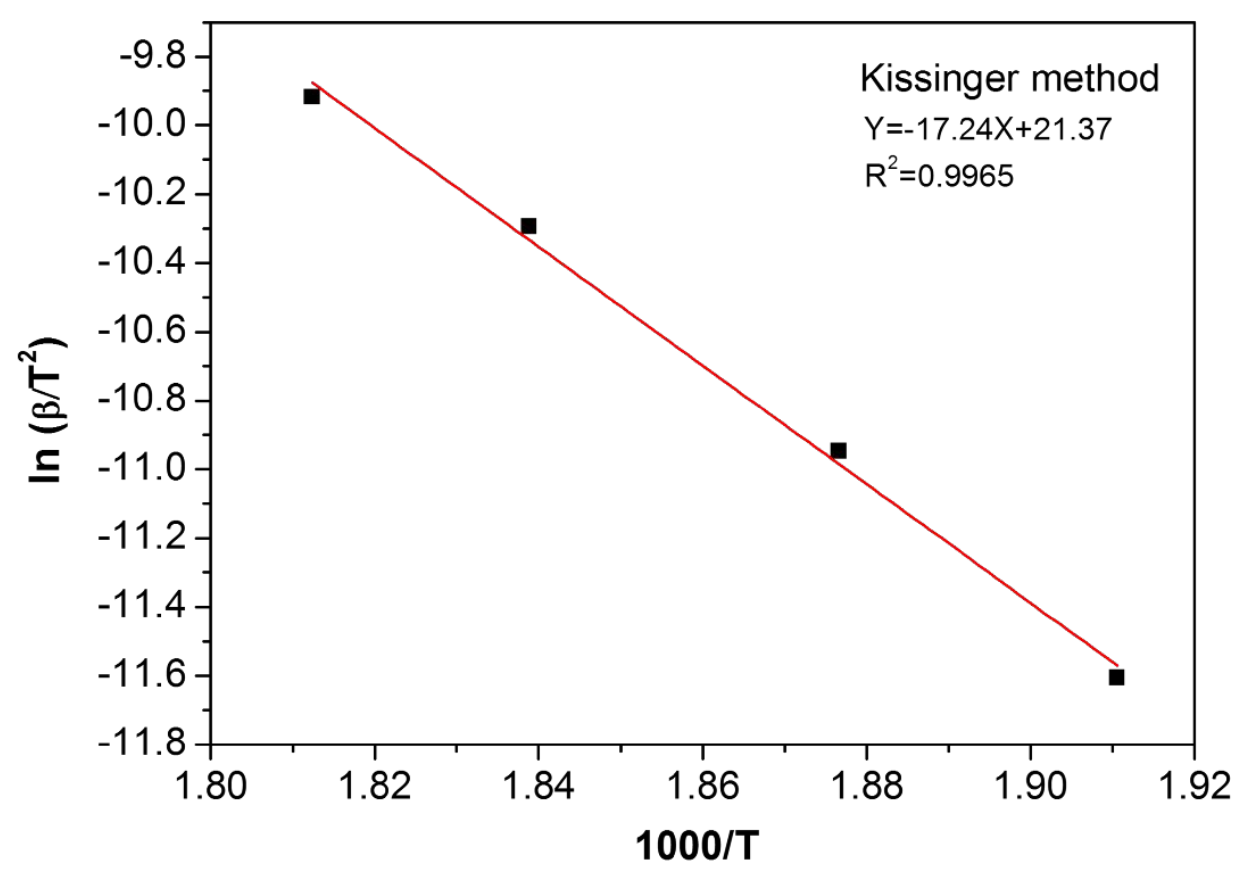

(a) Kinetic plot for DNPPc by Kissinger method

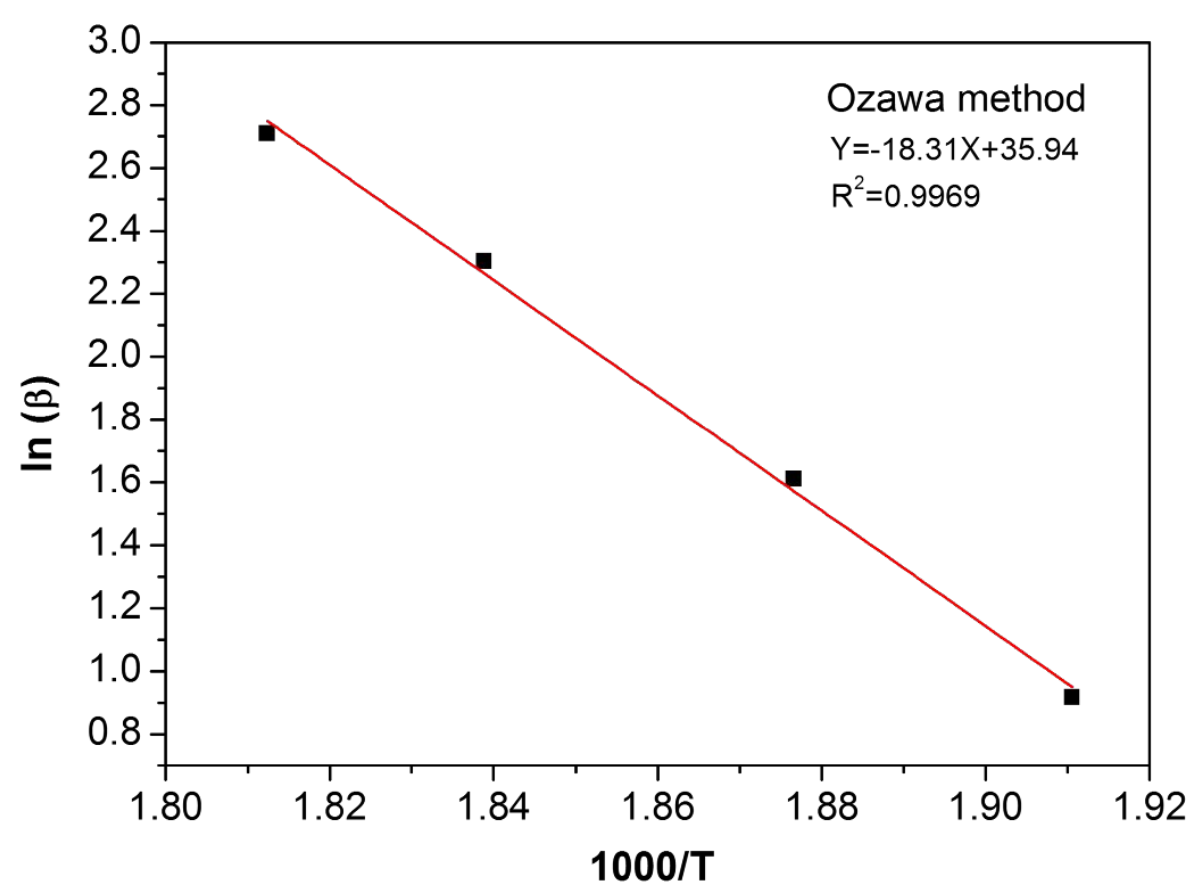

(b) Kinetic plot for DNPPc by Ozawa method 
Figure S5 Kinetic plots for DNPPc by (a) Kissinger method and (b) Ozawa method.

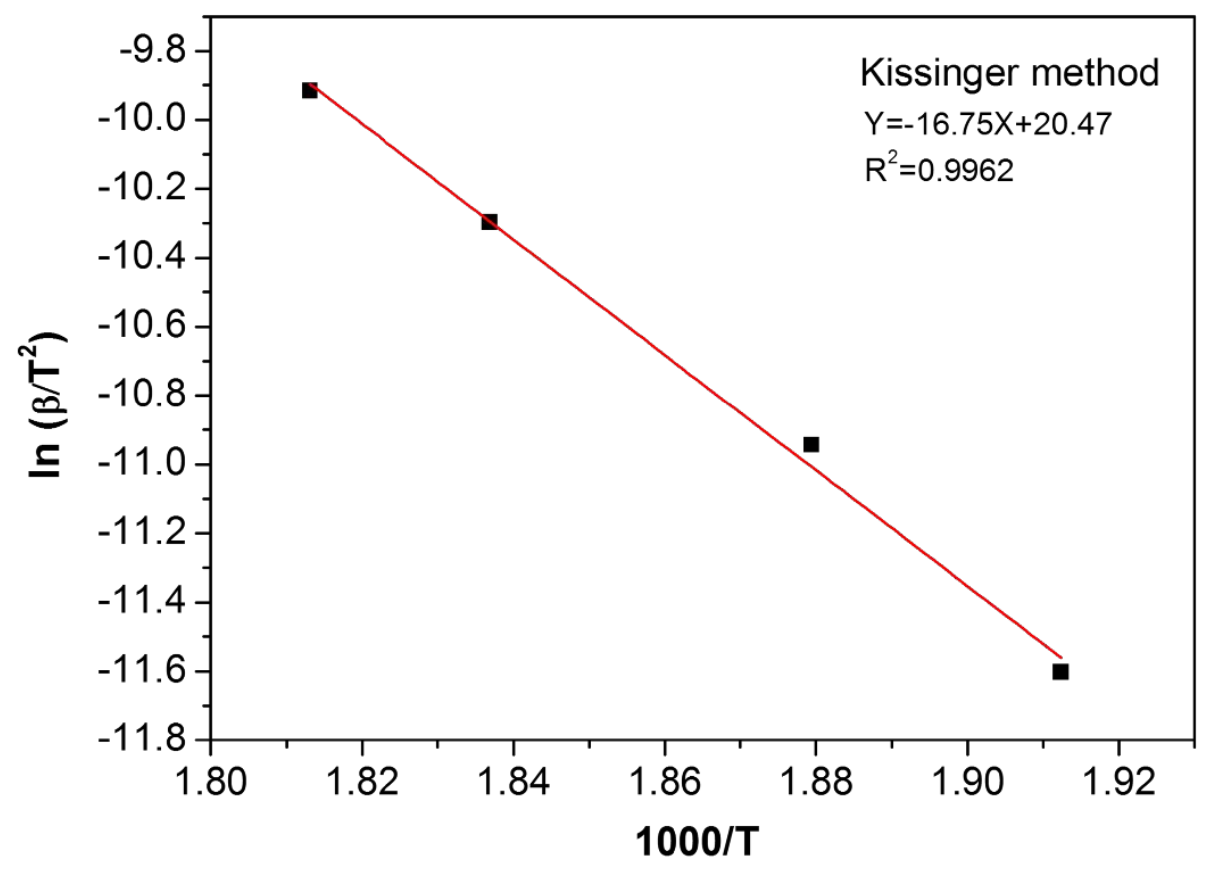

(a) Kinetic plot for DNPBc by Kissinger method

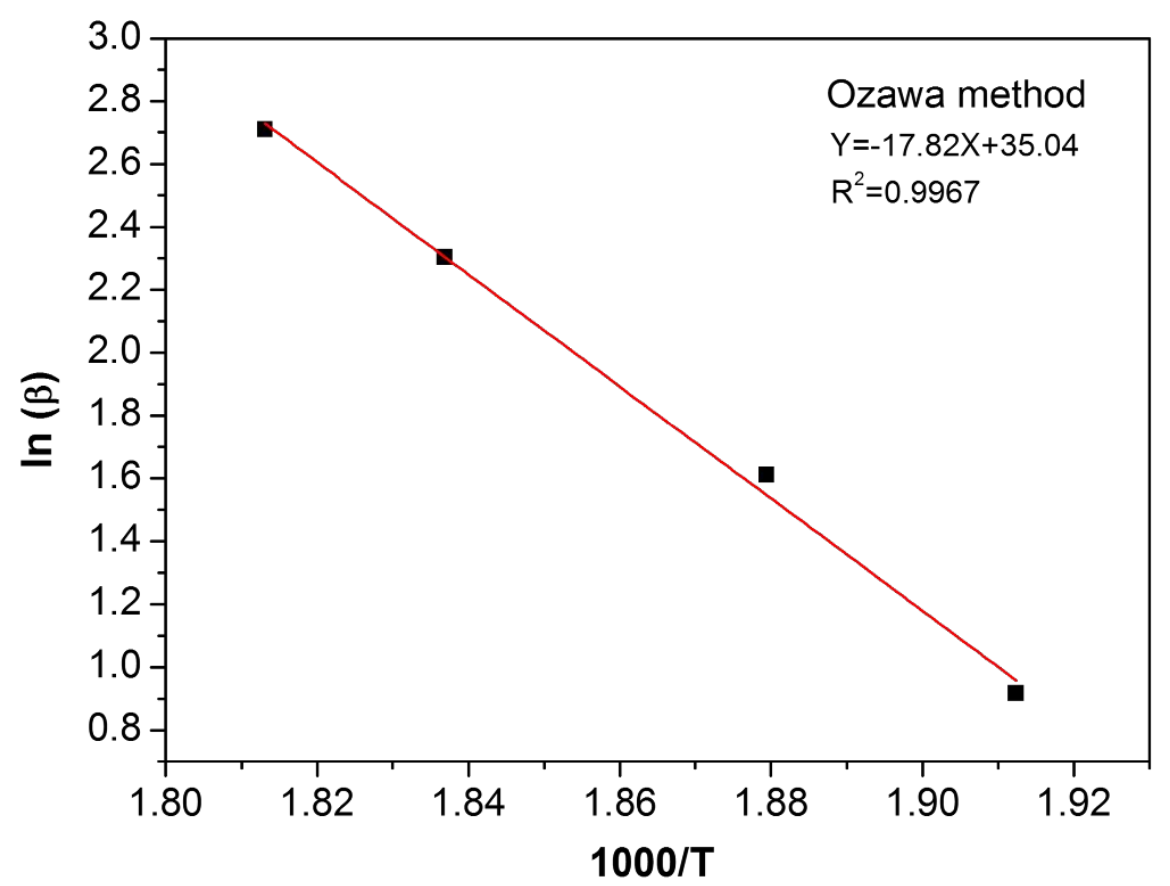

(b) Kinetic plot for DNPBc by Ozawa method 
Figure S6 Kinetic plots for DNPBc by (a) Kissinger method and (b) Ozawa method. 
Table S1 Thermal decomposition kinetic parameters of DNPAc

\begin{tabular}{ccccccc}
\hline$\beta\left({ }^{\circ} \mathrm{C} \cdot \mathrm{min}^{-1}\right)$ & $\mathrm{T}_{\mathrm{P}}\left({ }^{\circ} \mathrm{C}\right)$ & $\begin{array}{c}E_{\mathrm{K}} \\
\left(\mathrm{kJ} \cdot \mathrm{mol}^{-1}\right)\end{array}$ & $\begin{array}{c}\ln A \\
\left(\mathrm{~s}^{-1}\right)\end{array}$ & $r_{\mathrm{K}}$ & $\begin{array}{c}E_{\mathrm{O}} \\
\left(\mathrm{kJ} \cdot \mathrm{mol}^{-1}\right)\end{array}$ & $\begin{array}{c}E_{\mathrm{Average}} \\
\left(\mathrm{kJ} \cdot \mathrm{mol}^{-1}\right)\end{array}$ \\
\hline 2.5 & 247.02 & & & & & \\
5 & 257.47 & & & & & \\
10 & 271.65 & $121.57 \pm 5.05$ & $26.13 \pm 1.16$ & 0.9966 & $124.08 \pm 5.02$ & 0.9971 \\
15 & 279.31 & & & & & $122.82 \pm 5.03$ \\
\hline
\end{tabular}

Note: The values of $E_{a}$ and $\ln A$ are provided at a $95 \%$ confidence level.

Table S2 Thermal decomposition kinetic parameters of DNPPc

\begin{tabular}{cccccc}
\hline$\beta\left({ }^{\circ} \mathrm{C} \cdot \mathrm{min}^{-1}\right)$ & $\mathrm{T}_{\mathrm{P}}\left({ }^{\circ} \mathrm{C}\right)$ & $\begin{array}{c}E_{\mathrm{K}} \\
\left(\mathrm{kJ} \cdot \mathrm{mol}^{-1}\right)\end{array}$ & $\begin{array}{c}\ln A \\
\left(\mathrm{~s}^{-1}\right)\end{array}$ & $r_{\mathrm{K}}$ & $\begin{array}{c}E_{\mathrm{O}} \\
E_{\mathrm{Average}} \\
\left(\mathrm{kJ} \cdot \mathrm{mol}^{-1}\right)\end{array}$ \\
\hline 2.5 & 250.26 & & & & \\
$r_{\mathrm{O}}$ & & & \\
& & & & \\
10 & 259.72 & $143.34 \pm 6.02$ & $31.16 \pm 1.37$ & 0.9965 & $144.80 \pm 5.97$ \\
15 & 270.67 & & & & \\
\hline
\end{tabular}

Note: The values of $E_{a}$ and $\ln A$ are provided at a $95 \%$ confidence level.

Table S3 Thermal decomposition kinetic parameters of DNPBc

\begin{tabular}{ccccccc}
\hline$\beta\left({ }^{\circ} \mathrm{C} \cdot \mathrm{min}^{-1}\right)$ & $\mathrm{T}_{\mathrm{P}}\left({ }^{\circ} \mathrm{C}\right)$ & $\begin{array}{c}E_{\mathrm{K}} \\
\left(\mathrm{kJ} \cdot \mathrm{mol}^{-1}\right)\end{array}$ & $\begin{array}{c}\ln A \\
\left(\mathrm{~s}^{-1}\right)\end{array}$ & $r_{\mathrm{K}}$ & $\begin{array}{c}E_{\mathrm{O}} \\
\left(\mathrm{kJ} \cdot \mathrm{mol}^{-1}\right)\end{array}$ & $\begin{array}{c}E_{\mathrm{Average}} \\
\left(\mathrm{kJ} \cdot \mathrm{mol}^{-1}\right)\end{array}$ \\
\hline 2.5 & 249.75 & & & & & \\
5 & 258.93 & & & & & \\
$139.23 \pm 6.06$ & $30.19 \pm 1.38$ & 0.9962 & $140.89 \pm 6.02$ & 0.9967 & $140.06 \pm 6.04$ \\
15 & 271.25 & & & & & \\
\hline
\end{tabular}

Note: The values of $E_{a}$ and $\ln A$ are provided at a $95 \%$ confidence level. 
Table S4 Mechanical sensitivities of GAP and its mixtures with DNEPs and BuNENA

\begin{tabular}{cc}
\hline Sample & $\mathrm{H}_{50} / \mathrm{cm}$ \\
\hline GAP & 42.5 \\
DNPAc+GAP(4:10) & 44.5 \\
DNPPc+GAP(4:10) & 47.0 \\
DNPBc+GAP(4:10) & 50.5 \\
BuNENA+GAP(4:10) & 48.5 \\
\hline
\end{tabular}

Note: $\mathrm{H}_{50}$ is the height for $50 \%$ probability of explosion with the unit of $\mathrm{cm}$.

Table S5 Viscosity results of DNEPs+GAP mixtures and GAP under different temperatures

\begin{tabular}{cccccc}
\hline \multirow{2}{*}{ Sample } & \multicolumn{5}{c}{ Viscosity/mPa·s } \\
\cline { 2 - 6 } GAP & $30^{\circ} \mathrm{C}$ & $40^{\circ} \mathrm{C}$ & $50^{\circ} \mathrm{C}$ & $60^{\circ} \mathrm{C}$ & $70^{\circ} \mathrm{C}$ \\
\hline DNPAc+GAP(1:10) & 5720 & 2740 & 1469 & 872 & 550 \\
DNPAc+GAP(2:10) & 1460 & 775 & 452 & 285 & 190 \\
DNPAc+GAP(3:10) & 975 & 540 & 319 & 205 & 139 \\
DNPAc+GAP(4:10) & 683 & 384 & 235 & 153 & 103 \\
DNPPc+GAP(1:10) & 2300 & 1177 & 667 & 408 & 266 \\
DNPPc+GAP(2:10) & 1339 & 695 & 397 & 243 & 158 \\
DNPPc+GAP(3:10) & 1285 & 690 & 403 & 235 & 155 \\
DNPPc+GAP(4:10) & 938 & 515 & 311 & 197 & 133 \\
DNPBc+GAP(1:10) & 2278 & 1163 & 658 & 403 & 263 \\
DNPBc+GAP(2:10) & 1384 & 744 & 437 & 274 & 182 \\
DNPBc+GAP(3:10) & 885 & 493 & 296 & 190 & 128 \\
DNPBc+GAP(4:10) & 640 & 364 & 223 & 146 & 99 \\
\hline
\end{tabular}


Table S6 Plasticizing efficiency of DNPAc, DNPPc and DNPBc for GAP

\begin{tabular}{cccc}
\hline Mixtures & $\mathrm{T}_{\mathrm{g} 1}-\mathrm{T}_{\mathrm{g} 2} /{ }^{\circ} \mathrm{C}$ & $\lambda$ & $\varepsilon$ \\
\hline DNPAc+GAP & 44.8 & 1.15 & 51.44 \\
DNPPc+GAP & 46.2 & 1.24 & 57.19 \\
DNPBc+GAP & 53.1 & 1.28 & 68.08 \\
\hline
\end{tabular}

Table S7 Composition of propellant formulations

\begin{tabular}{|c|c|c|c|c|c|}
\hline \multicolumn{5}{|c|}{ Composition } & \multirow{2}{*}{ Ingredient $/ w t \%$} \\
\hline 1 & 2 & 3 & 4 & 5 & \\
\hline GAP & GAP & GAP & GAP & GAP & 8 \\
\hline HMX & HMX & HMX & HMX & HMX & 38 \\
\hline $\mathrm{Al}$ & $\mathrm{Al}$ & $\mathrm{Al}$ & $\mathrm{Al}$ & $\mathrm{Al}$ & 17 \\
\hline AP & $\mathrm{AP}$ & AP & $\mathrm{AP}$ & AP & 27 \\
\hline BuNENA & BDNPF/A & DNPAc & DNPPc & DNPBc & 10 \\
\hline
\end{tabular}

